\title{
Acceptability of bone antiresorptive therapy among HIV-infected adults at different stages of antiretroviral therapy
}

This article was published in the following Dove Press journal:

Patient Preference and Adherence

24 September 2014

Number of times this article has been viewed

\author{
Jillian Taras' \\ Gordon Arbess ${ }^{1,2}$ \\ James Owen ${ }^{1,2}$ \\ Charlie B Guiang ${ }^{1,2}$ \\ Darrell H S Tan ${ }^{1,3}$
}

'Faculty of Medicine, University of Toronto, Toronto, ON, Canada; ${ }^{2}$ Department of Family Medicine, St Michael's Hospital, Toronto, ON, Canada; ${ }^{3}$ Division of Infectious Diseases, St Michael's Hospital, Toronto, ON, Canada
Correspondence: Darrell H S Tan St Michael's Hospital, 30 Bond St, 4CC - Room 4-179,

Toronto ON, M5B IW8

Tel +l 4168645568

Fax +I 4168645310

Email darrell.tan@gmail.com
Purpose: Both HIV infection and antiretroviral therapy (ART) are associated with significant decreases in bone mineral density (BMD) and increased fracture rates. To prepare for a randomized controlled trial of prophylactic bone antiresorptive therapy during ART initiation, we assessed the acceptability of this strategy, bone health knowledge, and fracture risk among HIV-infected adults.

Methods: HIV-infected adults with no history of osteoporosis were recruited from one tertiary and one primary care HIV clinic. Participants completed a questionnaire and underwent chart review. The primary outcome was the proportion of respondents expressing interest in taking prophylactic bone antiresorptive therapy in conjunction with ART.

Results: Of 112 respondents, $25.0 \%$ were ART naïve, $23.2 \%$ had been taking ART for $\leq 1$ year, and $51.8 \%$ had been taking ART for $>1$ year. Half (51.9\%) indicated interest in taking shortcourse prophylactic bone antiresorptive therapy; this did not differ by ART status $(53.6 \%$ among ART-naïve, $51.3 \%$ among ART-treated; $P=0.84$, chi-square test). In exploratory multivariable analysis adjusted for ART status, a greater number of pills taken per day was positively associated with this outcome (adjusted odds ratio [OR] $=1.12$ per pill, 95\% confidence limit $[C L]=1.01,1.25$ ), while male sex was inversely associated (adjusted $\mathrm{OR}=0.05,95 \% \mathrm{CL}=0.01$, 0.24). Among those willing to take therapy, most (80.4\%) were willing to do so for "as long as needed" and preferred weekly dosing (70.9\%) to daily dosing (12.7\%).

Conclusions: Half of this sample would be willing to take bone antiresorptive therapy together with ART, with preferences for weekly dosing and for whatever duration may be required. These data will inform the design of future trials to protect bone health in HIV.

Keywords: HIV, bone antiresorptive therapy, prophylaxis

\section{Background}

With the advent of combination antiretroviral therapy (ART) for HIV infection, new long-term metabolic complications have emerged. Among these complications are decreases in bone mineral density (BMD) and the associated increased risks of fracture. A meta-analysis suggests that HIV patients are 6.4 times more likely to have low BMD, and 3.7 times more likely to have osteoporosis, than HIV-uninfected controls. ${ }^{1}$ Other studies have documented increasing rates of fractures in the setting of HIV, with an overrepresentation of fractures at fragility sites such as the vertebrae, hip, and wrist. ${ }^{2-4}$

The etiology of these problems is likely multifactorial, including an overrepresentation of traditional osteoporosis risk factors including smoking, hypogonadism, low body mass index, and excess alcohol consumption, a contribution from HIV itself, and an important iatrogenic impact of ART. ${ }^{5,6}$ Indeed, clinical trials of firstline 
ART among treatment-naïve HIV patients have consistently demonstrated dramatic net BMD decreases of $2 \%-6 \%$ during the first year after ART initiation. ${ }^{7,8}$ At present, however, the standard approach to bone health in HIV has typically ignored this critical time window. Current clinical guidelines and expert recommendations are simply to monitor for the development of osteoporosis by assessing BMD in patients who have accumulated enough clinical risk for fracture over time. $^{9-11}$

However, giving short-course bone antiresorptive therapy around the time of ART initiation is intuitively much more attractive than waiting for osteoporosis to develop in HIVinfected patients. Since BMD typically stabilizes after the first year on ART, a short course (eg, 6-12 months) of prophylaxis may be sufficient and could further avoid the costs, inconveniences, and toxicities of long-term osteoporosis treatment. There are no published data on the preventative use of such medications in the context of HIV. To prepare for a randomized controlled trial of prophylactic bone antiresorptive therapy during ART initiation, we assessed the acceptability of this strategy, as well as bone health knowledge and fracture risk, among HIV-infected adults.

\section{Methods}

We conducted a cross-sectional survey and chart review among patients attending routine clinic visits at a tertiary care HIV clinic and an academic primary care clinic in Toronto, Canada. Sequentially identified HIV-infected adults aged $\geq 18$ years with no known history of osteoporosis and no use of antiresorptive therapy were eligible.

The survey instrument was a self-administered 18-item questionnaire addressing three domains: patient preferences regarding bone antiresorptive therapy, knowledge about bone health, and fracture risk. The questionnaire was developed as follows. Item generation and item reduction were completed by the study team after literature review, with several items modified from previously published papers and/or validated scales. ${ }^{12-14}$ The first five participants served as pilot participants, allowing assessment of administration logistics and understandability; no issues were identified requiring modification of the survey instrument. Permission was also sought to extract information about participants' demographics, HIV history, and fracture risk from clinic charts. Fracture risk was objectively quantified using the World Health Organization Fracture Risk Assessment Tool $\left(\right.$ FRAX $\left.^{\circledR}\right)$, validated for Canadian populations. ${ }^{15}$

The primary outcome measure was the proportion of respondents answering "yes" in response to the question, "If you were asked today, would you be interested in taking an osteoporosis prevention medication, together with anti-HIV medications?" This question was preceded by the following short, plain-language statement about ART and osteoporosis prevention medications: "Anti-HIV medications are extremely important for treating HIV infection. However, they can sometimes cause side effects, including weakening of the bones, that could increase the risk of osteoporosis and rarely, lead to fractures. Safe, effective medications exist that can decrease the risk of osteoporosis and fractures. However, these osteoporosis prevention medications are not routinely used in HIV-infected persons unless there is a proven problem with the person's bone health." Because the current use of ART may influence participants' willingness to take bone antiresorptive therapy, the primary outcome was calculated separately for two strata: patients naïve to ART and those already on ART.

Secondary outcome measures included participants' preferences regarding the potential use of antiresorptive therapy, knowledge regarding bone health, subjective assessment of their fracture risk, and objective fracture risk as evaluated using the FRAX ${ }^{\circledR}$ tool. Exploratory univariable and multivariable logistic regression modeling was used to identify predictors of participants' willingness to use antiresorptive therapy. Variables were included in the multivariable model if they produced changes of $\geq 10 \%$ in the parameter estimate for the primary predictor variable of interest, ART status, and were not collinear with other variables.

The sample size was based on the minimum number of participants required to estimate the proportion who would be willing to take prophylactic bone antiresorptive therapy during ART initiation with a reasonable level of precision. Using the equation $\mathrm{N}=\mathrm{Z}_{1-\alpha / 2}^{2} * \mathrm{p}(1-\mathrm{p}) / l^{2}$, where $\mathrm{Z}_{1-\alpha / 2}$ is the $1-\alpha / 2$ critical value of the standard normal distribution for $\alpha=0.05$, $\mathrm{p}$ is the proportion of interest which was conservatively set at 0.5 , and $l$ is half the length of the desired $95 \%$ confidence interval, and allowing for $10 \%$ incomplete responses, we calculated that a sample of 106 participants would allow estimation of the true prevalence with reasonable precision $(l=0.1)$. We aimed for half of the recruited sample to be either ART-naïve or within the first year of ART initiation.

Approval was obtained from the St. Michael's Hospital Research Ethics Board prior to conducting any study activities.

\section{Results}

Between July and August 2013, 119 sequential patients were asked to participate, of whom two declined without 


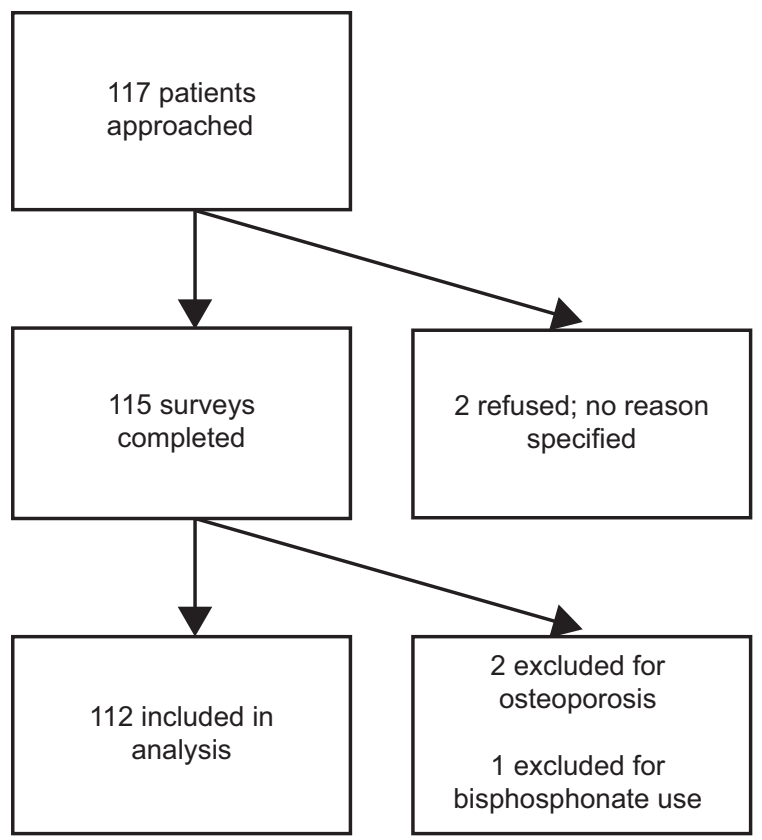

Figure I Flowchart of participants approached and included.

specifying a reason. Of the 117 surveys that were returned, two were excluded due to a history of osteoporosis and one was removed due to concurrent use of a bisphosphonate (Figure 1). Of the 112 respondents included in the final analysis, median (interquartile range, IQR) age was 43 (36-51) years and $89(78.8 \%)$ were male. Roughly half $(51.8 \%)$ had been taking ART for $>1$ year, $23.2 \%$ had been taking ART for $\leq 1$ year, and $25.0 \%$ were ART naïve. Additional participant characteristics are shown in Table 1.

\section{Preferences regarding bone antiresorptive therapy}

In the primary analysis, half (51.9\%) of participants indicated interest in taking prophylactic bone antiresorptive therapy in conjunction with ART, and this proportion did not differ by ART treatment status (53.6\% among ART-naïve, $51.3 \%$ among ART-treated; $P=0.84$, chi-square test). Male sex was inversely associated with this outcome in both univariable (odds ratio $[\mathrm{OR}]=0.07,95 \%$ confidence limit $[\mathrm{CL}]=0.02,0.33$ ) and multivariable (adjusted $\mathrm{OR}=0.05$, $95 \% \mathrm{CL}=0.01,0.24$ ) analysis (Table 2 ). The only other participant characteristic significantly associated with willingness to use antiresorptive therapy was taking a greater number of pills per day (adjusted OR $=1.12$ per pill, $95 \%$ $\mathrm{CL}=1.01,1.25$ ).

Among those willing to take therapy, most (80.4\%) were willing to do so for "as long as needed", and preferred weekly dosing $(70.9 \%)$ to daily dosing $(12.7 \%)$.
Table I Respondent characteristics

\begin{tabular}{|c|c|}
\hline Variable & Value $^{a}$ \\
\hline \multicolumn{2}{|l|}{ Sex } \\
\hline Male & $89(78.8)$ \\
\hline Female & $23(21.2)$ \\
\hline Other & 0 \\
\hline Age & $43(36-5 I)$ \\
\hline \multicolumn{2}{|l|}{ Race } \\
\hline Caucasian & $47(42.0)$ \\
\hline Black & $34(30.4)$ \\
\hline Asian & $12(10.1)$ \\
\hline Other & $17(15.8)$ \\
\hline \multicolumn{2}{|l|}{ ART use } \\
\hline ART naïve & $28(25.0)$ \\
\hline Initiated ART within 12 months & $26(23.2)$ \\
\hline Initiated ART $>12$ months & $58(51.8)$ \\
\hline \multicolumn{2}{|l|}{ HIV risk factors } \\
\hline MSM & $61(54.5)$ \\
\hline IDU & $2(1.8)$ \\
\hline Blood product & $4(3.6)$ \\
\hline Heterosexual & $39(34.8)$ \\
\hline Other/unknown & $6(5.4)$ \\
\hline Years since HIV diagnosis & $8(3-12)$ \\
\hline Current CD4 count (cells $/ \mathrm{mm}^{3}$ ) & $477(297-6 \mid 5)$ \\
\hline Nadir CD4 count (cells/mm³) & $207(94-380)$ \\
\hline \multicolumn{2}{|c|}{ Viral load undetectable ( $<40$ copies $/ \mathrm{mL}$ ) } \\
\hline ART naïve & $2(7.1)$ \\
\hline Initiated ART within 12 months & $12(46.2)$ \\
\hline Initiated ART $>12$ months & $54(93.1)$ \\
\hline \multicolumn{2}{|l|}{ Fracture history } \\
\hline No previous fracture & $86(78.9)$ \\
\hline Previous facture & $23(21.1)$ \\
\hline \multicolumn{2}{|l|}{ Previous bone mineral density test } \\
\hline Yes & $12(10.7)$ \\
\hline No & $100(89.3)$ \\
\hline \multicolumn{2}{|l|}{ Vitamin D use } \\
\hline Yes & $28(25.0)$ \\
\hline No & $84(75.0)$ \\
\hline \multicolumn{2}{|c|}{$\begin{array}{l}\text { Problems with history of heartburn, reflux, } \\
\text { or stomach acid }\end{array}$} \\
\hline Yes & $39(35.1)$ \\
\hline No & $72(64.9)$ \\
\hline
\end{tabular}

Notes: ${ }^{\mathrm{a}}$ Values shown are median (interquartile range) and frequency (percentage). ${ }^{b}$ One participant did not respond to this question.

Abbreviations: ART, antiretroviral therapy; IDU, injection drug user; MSM, man who has sex with men.

\section{Knowledge regarding bone health}

Respondents' knowledge surrounding bone health and HIV was modest. Most participants (59.8\%) provided a correct understanding of what the term "osteoporosis" means in response to an open-ended question. However, in a series of true/false questions, roughly one third to one half of respondents were unaware that HIV (44.4\%), HIV medications (50.5\%), smoking (35.6\%), and alcohol consumption (32.7\%) increase the risk of fracture, and fully $83.2 \%$ of respondents 
Table 2 Characteristics associated to willingness to take antiresorptive therapy

\begin{tabular}{|c|c|c|c|c|}
\hline \multirow[t]{2}{*}{ Variable } & \multicolumn{2}{|l|}{ Univariable model } & \multicolumn{2}{|l|}{ Multivariable model } \\
\hline & Odds ratio $(95 \% \mathrm{CL})$ & $P$-value & Odds ratio $(95 \% \mathrm{CL})$ & $P$-value \\
\hline Male & $0.07(0.02,0.33)$ & 0.0006 & $0.05(0.01,0.24)$ & 0.0003 \\
\hline \multicolumn{5}{|l|}{ ART status } \\
\hline ART naïve & 1.00 & & & \\
\hline Initiated ART within 12 months & $1.67(0.66,4.22)$ & 0.28 & & \\
\hline Initiated ART $>12$ months & $0.84(0.34,2.08)$ & 0.57 & & \\
\hline Undetectable viral load ( $<40$ copies $/ \mathrm{mL})$ & $0.6 \mathrm{I}(0.28, \mathrm{I} .33)$ & 0.15 & & \\
\hline Number of medications/day & $0.94(0.85,1.05)$ & 0.26 & & \\
\hline Number of pills/day ${ }^{a}$ & $0.94(0.87,1.02)$ & 0.12 & $1.12(1.01,1.25)$ & 0.03 \\
\hline Prior fracture & $1.34(0.52,3.49)$ & 0.55 & $0.43(0.13,1.42)$ & 0.17 \\
\hline Number of osteoporotic risk factors & $0.89(0.54,1.47)$ & 0.66 & $0.47(0.20,1.12)$ & 0.09 \\
\hline Knows definition of osteoporosis & $0.83(0.38,1.80)$ & 0.63 & & \\
\hline
\end{tabular}

Note: aNumber of pills/day refers to all medications taken by patient.

Abbreviations: ART, antiretroviral therapy; $\mathrm{CL}$, confidence limit.

erroneously believed that "osteoporosis is something that only matters for the elderly".

\section{Fracture risk}

Respondents were asked to predict their likelihood of fracture in the next 10 years both qualitatively (low, medium, or high risk) and quantitatively (by indicating a percentage chance). Most respondents (58.2\%) felt themselves to be at low risk, while $35.5 \%$ estimated medium and $6.36 \%$ high risk. Respondents' quantitative estimations of risk in general matched their qualitative perceptions of risk, but at an order of magnitude higher than standard definitions (low $<10 \%$, medium 10\%-20\%, high >20\%) (Figure 2).

Objective fracture risk assessment using the FRAX ${ }^{\circledR}$ tool validated for Canadian populations corroborated the overall low fracture risk of this sample at the time of study. Median (IQR) risk of a major osteoporotic risk factor within 10 years was $1.9 \%(1.3 \%-3.6 \%)$, with only two

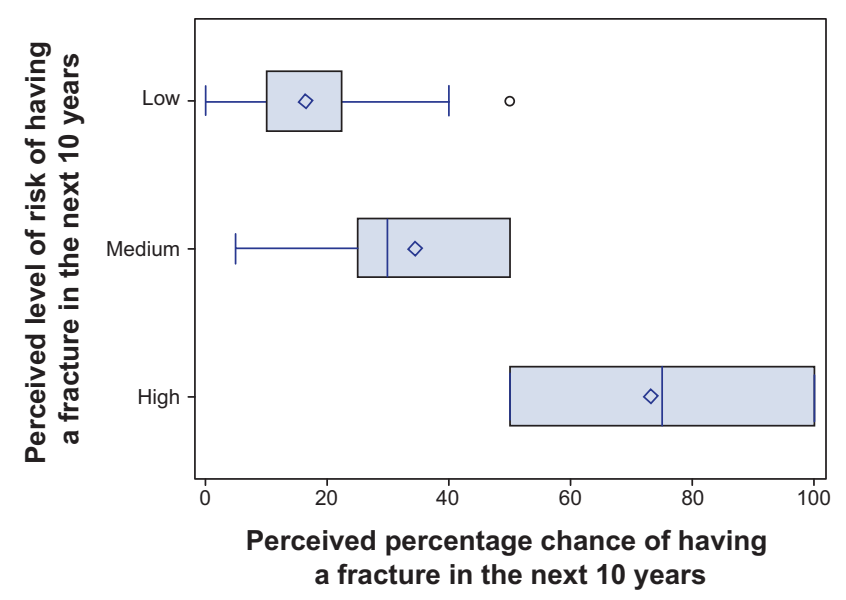

Figure 2 Perceived definitions of fracture risk. Notes: The diamond represents the mean. The circle represents an outlier. participants at medium risk ( $10 \%$ and $15 \%$ respectively). Risk for an osteoporotic hip fracture was still lower, at $0.1 \%$ $(0.0 \%-0.3 \%)$.

While $64.5 \%$ of respondents reported having no osteoporosis risk factors, $24.3 \%$ reported one, $7.5 \%$ reported two, and 3.7\% reported three, including cigarette smoking $(n=25)$, previous or current corticosteroid use $(n=9)$, parental hip fracture $(n=7)$, excess alcohol intake ( $\geq 3$ drinks/day) $(n=6)$, rheumatoid arthritis $(n=5)$, chronic liver disease $(n=3)$, and menopause before the age of 45 years $(n=2)$. The one "protective" variable studied was regular (once a week or more) weightlifting exercise or resistance training, reported by $53.6 \%$.

\section{Discussion}

To prepare for a randomized controlled trial, we assessed the acceptability of prophylactic bone antiresorptive therapy during ART initiation among HIV-infected adults. Our finding that half of respondents would be willing to take such therapy, in most cases for whatever duration may be required, supports the feasibility and acceptability of conducting such a trial.

Given participants' preferences for weekly dosing and the modest prevalence of "heartburn" in this population, alendronate would be an attractive option for future study. An extensive literature in HIV-uninfected populations demonstrates that alendronate is efficacious in preventing BMD loss in postmenopausal women, ${ }^{16,17}$ patients taking glucocorticoids, ${ }^{18,19}$ and men receiving androgen deprivation therapy for prostate cancer, ${ }^{20,21}$ for up to 4 years of follow-up. Further, this drug has been safely and effectively used to improve BMD in HIV-infected persons with established osteopenia or osteoporosis. ${ }^{22-24}$ Longer-acting agents such as a 
single dose of zoledronate and osteoporosis medications from different pharmacological classes may also warrant study.

That males in this sample were less willing to use bone antiresorptive therapy than females is perhaps not surprising, given lay perceptions of osteoporosis as a predominantly women's health issue and given that women's BMD declines at a faster rate than men, particularly after menopause, ${ }^{6}$ future work should seek to increase awareness of the importance of bone health among men living with HIV. Taking more pills per day was positively associated with this outcome, perhaps reflecting trepidation regarding pill-taking among treatment-naïve patients and familiarity with pill-taking among those already on ART. In addition, overestimation of fracture risk was common, with $35.5 \%$ and $6.4 \%$ of the sample subjectively perceiving their fracture risk as "medium" and "high", respectively, despite a low median objective fracture risk of $1.9 \%$. Together with the modest level of bone health knowledge we observed, these findings highlight the importance of patient counseling about this mounting health issue in HIV.

This study has limitations that warrant consideration. First, although it was encouraging that female sex was positively associated with interest in bone antiresorptive therapy, only $21.2 \%$ of respondents were female. Future studies should assess the preferences of HIV-infected women regarding bone health in greater detail. Second, we relied on self-report for most osteoporosis risk factors, and respondents' misinterpretation of some items (eg, mistaking osteoarthritis for rheumatoid arthritis, etc) may have resulted in some misclassification. However, this possibility is unlikely to change our overall conclusions regarding the magnitude of fracture risk in this sample. Third, although validated for Canadian populations, the $\mathrm{FRAX}^{\circledR}$ tool does not incorporate HIV status, which is likely an independent risk factor for fracture; hence, participants' true fracture risks may have been underestimated. Fourth, framing the osteoporosis prevention medications as "safe and effective" may have biased towards a greater degree of acceptability. Finally, the total number of respondents was modest, and recruitment was limited to two academic centers. However, we included both a primary and a tertiary care site to increase generalizability, and we surpassed our target sample size.

Importantly, the low overall fracture risk in this sample does not mean that fracture risk in HIV is less important than epidemiological data on osteoporosis prevalence would otherwise suggest. Indeed, as HIV treatment guidelines worldwide increasingly move towards the earlier initiation of ART in HIV-infected persons, patients' cumulative exposure to this long-term, partially iatrogenic health issue will only increase. Given the striking and predictable decreases in BMD during the first year of ART, short-course bone antiresorptive therapy at this time is an attractive approach warranting further study. Our findings support the acceptability of future clinical trials in this area.

\section{Disclosure}

Darrell H S Tan is supported by an RCT Mentoring Award from the Canadian Institutes of Health Research. The authors report no other conflicts of interest in this work.

\section{References}

1. Brown TT, Qaqish RB. Antiretroviral therapy and the prevalence of osteopenia and osteoporosis: a meta-analytic review. AIDS. 2006;20(17):2165-2174.

2. Triant VA, Brown TT, Lee H, Grinspoon SK. Fracture prevalence among human immunodeficiency virus (HIV)-infected versus non-HIVinfected patients in a large U.S. healthcare system. J Clin Endocrinol Metab. 2008;93(9):3499-3504.

3. Bedimo R, Maalouf NM, Zhang S, Drechsler H, Tebas P. Osteoporotic fracture risk associated with cumulative exposure to tenofovir and other antiretroviral agents. AIDS. 2012;26(7):825-831.

4. Young B, Dao CN, Buchacz K, Baker R, Brooks JT; HIV Outpatient Study (HOPS) Investigators. Increased rates of bone fracture among HIV-infected persons in the HIV Outpatient Study (HOPS) compared with the US general population, 2000-2006. Clin Infect Dis. 2011; 52(8):1061-1068.

5. Amorosa V, Tebas P. Bone disease and HIV infection. Clin Infect Dis. 2006;42:108-14.

6. Thomas J, Doherty SM. HIV infection - a risk factor for osteoporosis J Acquir Immune Defic Syndr. 2003;33:281-91.

7. McComsey GA, Kitch D, Daar ES, et al. Bone mineral density and fractures in antiretroviral-naive persons randomized to receive abacavirlamivudine or tenofovir disoproxil fumarate-emtricitabine along with efavirenz or atazanavir-ritonavir: Aids Clinical Trials Group A5224s, a substudy of ACTG A5202. J Infect Dis. 2011;203(12):1791-1801.

8. Stellbrink HJ, Orkin C, Arribas JR, et al; ASSERT Study Group. Comparison of changes in bone density and turnover with abacavir-lamivudine versus tenofovir-emtricitabine in HIV-infected adults: 48-week results from the ASSERT study. Clin Infect Dis. 2010;51(8): 963-972.

9. Papaioannou A, Morin S, Cheung AM, et al; Scientific Advisory Council of Osteoporosis Canada. 2010 clinical practice guidelines for the diagnosis and management of osteoporosis in Canada: summary. CMAJ. 2010;182(17):1864-1873.

10. McComsey GA, Tebas P, Shane E, et al. Bone disease in HIV infection: a practical review and recommendations for HIV care providers. Clin Infect Dis. 2010;51(8):937-946.

11. Aberg JA, Gallant JE, Ghanem KG, Emmanuel P, Zingman BS, Horberg MA. Primary care guidelines for the management of persons infected with HIV: 2013 update by the HIV Medicine Association of the Infectious Diseases Society of America. Clin Infect Dis. 2014;58(1): $1-10$.

12. Simon JA, Lewiecki EM, Smith ME, Petruschke RA, Wang L, Palmisano JJ. Patient preference for once-weekly alendronate $70 \mathrm{mg}$ versus once-daily alendronate $10 \mathrm{mg}$ : a multicenter, randomized, openlabel, crossover study. Clin Ther. 2002;24(11):1871-1886.

13. Douglas F, Petrie KJ, Cundy T, Horne A, Gamble G, Grey A. Differing perceptions of intervention thresholds for fracture risk: a survey of patients and doctors. Osteoporos Int. 2012;23(8):2135-2140. 
14. Ailinger RL, Harper DC, Lasus HA. Bone up on osteoporosis. Development of the Facts on Osteoporosis Quiz. Orthop Nurs. 1998;17(5): 66-73.

15. FRAX WHO Fracture Risk Assessment Tool [Internet]. University of Sheffield: World Health Organization Collaborating Center for Metabolic Bone Disease; c2011. Available at: http://www.shef.ac.uk/ FRAX/tool.aspx? country=19. Accessed August 11, 2014.

16. McClung MR, Wasnich RD, Hosking DJ, et al; Early Postmenopausal Intervention Cohort Study. Prevention of postmenopausal bone loss: six-year results from the Early Postmenopausal Intervention Cohort Study. J Clin Endocrinol Metab. 2004;89(10):4879-4885.

17. Ravn P, Bidstrup M, Wasnich RD, et al. Alendronate and estrogenprogestin in the long-term prevention of bone loss: four-year results from the early postmenopausal intervention cohort study. A randomized, controlled trial. Ann Intern Med. 1999;131(12):935-942.

18. Stoch SA, Saag KG, Greenwald M, et al. Once-weekly oral alendronate $70 \mathrm{mg}$ in patients with glucocorticoid-induced bone loss: a 12-month randomized, placebo-controlled clinical trial. J Rheumatol. 2009;36(8): $1705-1714$.

19. Adachi JD, Saag KG, Delmas PD, et al. Two-year effects of alendronate on bone mineral density and vertebral fracture in patients receiving glucocorticoids: a randomized, double-blind, placebo-controlled extension trial. Arthritis Rheum. 2001;44(1):202-211.
20. Greenspan SL, Nelson JB, Trump DL, Resnick NM. Effect of onceweekly oral alendronate on bone loss in men receiving androgen deprivation therapy for prostate cancer: a randomized trial. Ann Intern Med. 2007;146(6):416-424.

21. Klotz LH, McNeill IY, Kebabdjian M, Zhang L, Chin JL; Canadian Urology Research Consortium. A phase 3, double-blind, randomised, parallel-group, placebo-controlled study of oral weekly alendronate for the prevention of androgen deprivation bone loss in nonmetastatic prostate cancer: the Cancer and Osteoporosis Research with Alendronate and Leuprolide (CORAL) study. Eur Urol. 2013;63(5):927-935.

22. McComsey GA, Kendall MA, Tebas P, et al. Alendronate with calcium and vitamin D supplementation is safe and effective for the treatment of decreased bone mineral density in HIV. AIDS. 2007;21(18): 2473-2482.

23. Mondy K, Powderly WG, Claxton SA, et al. Alendronate, vitamin D, and calcium for the treatment of osteopenia/osteoporosis associated with HIV infection. J Acquir Immune Defic Syndr. 2005;38(4):426-431.

24. Guaraldi G, Orlando G, Madeddu G, et al. Alendronate reduces bone resorption in HIV-associated osteopenia/osteoporosis. HIV Clin Trials. 2004;5(5):269-277.
Patient Preference and Adherence

\section{Publish your work in this journal}

Patient Preference and Adherence is an international, peer-reviewed, open access journal that focuses on the growing importance of patient preference and adherence throughout the therapeutic continuum. Patient satisfaction, acceptability, quality of life, compliance, persistence and their role in developing new therapeutic modalities and compounds to optimize

\section{Dovepress}

clinical outcomes for existing disease states are major areas of interest for the journal. This journal has been accepted for indexing on PubMed Central. The manuscript management system is completely online and includes a very quick and fair peer-review system, which is all easy to use. Visit http://www. dovepress.com/testimonials.php to read real quotes from published authors. 